used to direct a systematic literature search. Published evidence was appraised using the GRADE system. Where the literature search identified sufficient evidence, the GDG made a guideline recommendation. Where there was insufficient evidence, the GDG drafted recommendations based on their expert opinion and reviewed these using a formal Delphi consensus process. This was a joint society (BSPED/CCLG) multidisciplinary national endeavour done to NICE methodology and overseen by RCPCH.

Results The literature search identified 568 articles covering the period Jan 1990 -March 2017. The most commonly reported causes of iTPS and iCDI in children are Langerhans Cell Hystiocytosis (LCH), Germ Cell Tumours (GCT) and craniopharingioma (CP). The average prevalence of LCH, GCT and $\mathrm{CP}$ in 11 case series (including 741 patients) is 16\%, $13 \%$ and $12 \%$, respectively. Overall, congenital defects are responsible for $19 \%$ of the iTPS/iCDI cases, whilst infectious diseases (2\%), trauma (1\%) and inflammatory/autoimmune conditions (1\%) rarely occur in children. In $29 \%$ of the cases no aetiology is identified. Causes of pituitary stalk lesions in adults, metastatic tumours and neurosarcoidosis, do not form part of the differential diagnosis in children. What constitutes a TPS is not consistently defined across studies. High quality evidence was lacking for the majority of the clinical questions and two rounds of Delphi consensus were undertaken. A decision-making flowchart has been developed and will accompany the guideline.

Conclusion The likely aetiology of iTPS and iCDI in children differs from that in adults and justifies the development of age appropriate decision making management guidelines to inform best practice nationally. This will form the basis for future audits of practice and outcomes and is intended to improve the quality of care of children and young people with iTPS and iCDI.

\section{G357 NATIONAL UK GUIDELINES FOR THE INVESTIGATION, TREATMENT AND LONG-TERM FOLLOW-UP OF PAEDIATRIC CRANIOPHARYNGIOMA}

\begin{abstract}
${ }^{1,2} \mathrm{HW}$ Gan, ${ }^{3} \mathrm{P}$ Morillon, ${ }^{4} \mathrm{C}$ Mallucci, ${ }^{5} \mathrm{~A}$ Gamble, ${ }^{6} \mathrm{~S}$ Wilne, ${ }^{7} \mathrm{~B}$ Harrison, ${ }^{2} \mathrm{H}$ Spoudeas. ${ }^{1}$ Genetics and Genomic Medicine Programme, University College London Great Ormond Street Institute of Child Health, London, UK; ${ }^{2}$ Department of Paediatric Endocrinology, Great Ormond Street Hospital for Children NHS Foundation Trust, London, UK; ${ }^{3}$ Department of Emergency Medicine, Kingston Hospital NHS Foundation Trust, London, UK; ${ }^{4}$ Department of Paediatric Neurosurgery, Alder Hey Children's NHS Foundation Trust, Liverpool, UK; ${ }^{5}$ Children's Cancer and Leukaemia Group, Leicester, UK; ${ }^{6}$ Nottingham Children's Hospital, Nottingham University Hospitals NHS Trust, Nottingham, UK; ${ }^{7}$ Department of Endocrine Surgery, Sheffield Teaching Hospitals NHS Foundation Trust, Sheffield, UK
\end{abstract}

10.1136/archdischild-2018-rcpch.347

Aims Although rare, craniopharyngiomas are the commonest suprasellar tumour in childhood. Despite high overall survival, children and young people $<19$ years with craniopharyngiomas are at risk of multiple relapses and long-term tumour- and treatment-related neuroendocrine, cognitive and visual morbidity. We sought to provide, for the first time, a national standard for best practice based on currently available evidence for the assessment, treatment and follow-up of paediatric craniopharyngiomas under the auspices of the RCPCH, UK Children's Cancer and Leukaemia Group (CCLG) and the British Society for Paediatric Endocrinology and Diabetes (BSPED).

Methods Clinical questions were formulated based on a PICO (Population, Intervention, Comparison, Outcome) format by a multidisciplinary Guideline Development Group. Systematic searches were conducted via the Ovid MEDLINE (1946-February 2017) and Cochrane Library (2016, Issue 12) databases, identifying 2023 separate research articles. Publications underwent a three-tier filtering process and 300 were reviewed using the GRADE approach. Where recommendations could not be made, a two-stage international Delphi consensus process was conducted.

Results 44 clinical questions were identified, leading to 35 recommendations largely based on low to very low quality evidence. 30 further recommendations achieved $>70 \%$ agreement via the Delphi consensus process. Important highlights include the recommendation that craniopharyngiomas are managed in tertiary paediatric centres with sufficient neuro-oncology, neurosurgery, endocrinology, radiology, pathology and neuropsychology multidisciplinary experience. At diagnosis, tumours should be graded using the 'Paris' grading system and subsequent surgical treatment tailored to avoid hypothalamic damage, with adjuvant upfront radiotherapy being offered where tumour resection is incomplete. Detailed recommendations on the neuroendocrine, ophthalmological and psychological pretreatment assessment of patients and long-term follow-up of survivors are also made, with a review on the safety of growth hormone replacement therapy in this cohort.

Conclusions These guidelines provide the first evidence- and consensus-based national recommendations for the management of paediatric craniopharyngioma, and highlight the need for further research in areas such as the efficacy of proton beam therapy, radiosurgery and intracystic therapies in children, and the management of late effects such as hypothalamic obesity. Through their implementation, we hope to achieve better consistency in the quality of care of such patients and improve long-term quality of survival.

\section{G358 MENTAL HEALTH NEEDS OF LONG TERM SURVIVORS OF CHILDHOOD AND YOUNG ADULT CANCER}

${ }^{1,2,3} \mathrm{AJ}$ Friend, ${ }^{1,3,4} \mathrm{AW}$ Glaser, ${ }^{2,4} \mathrm{RG}$ Feltbower. 'Leeds Institute of Cancer and Pathology, University of Leeds, Leeds, UK; ${ }^{2}$ Division of Epidemiology and Biostatistic, University of Leeds, Leeds, UK; ${ }^{3}$ Department of Paediatric Oncology, Leeds Children's Hospital, Leeds, UK; ${ }^{4}$ Leeds Institute of Data Analytics, University of Leeds, Leeds, UK

\subsection{6/archdischild-2018-rcpch.348}

Background/objectives Survivors of children's and young people's cancer are known to have an increased risk of cognitive difficulties compared to the general population, however less is known about emotional and behavioural problems. A recent systematic review highlighted the lack of consensus regarding the psychiatric needs of these patients. We aimed to further explore the prevalence of psychiatric disorder in long-term survivors of children's and young people's cancer.

Methods Cancer registration records from a regional population-based registry of children's and young people's cancer in Yorkshire were electronically linked with the Hospital Episode Statistics Mental Health and Learning Disabilities Data Set (MHLDDS) covering all admissions in England between 2005 and 2016. The analysis was limited to those aged 0-29 years at diagnosis between 1974 and 2012; we excluded those diagnosed less than 5 years ago to ensure that patients who required psychological support during their acute treatment were not included.

Results We had registry data for 8092 patients who had survived a minimum of 5 years following a diagnosis of 